Archives de sciences sociales des religions

139 | juillet - sepembre 2007

Prophètes, messages et médias

Ahmed Deedat et l'islam indien en Afrique du Sud Individualisme et transnationalisme islamique

Samadia Sadouni

\title{
CpenEdition
}

Journals

Édition électronique

URL : http://journals.openedition.org/assr/9123

DOI : 10.4000/assr.9123

ISSN : $1777-5825$

Éditeur

Éditions de l'EHESS

Édition imprimée

Date de publication : 1 septembre 2007

Pagination : 101-118

ISBN : 978-2-7132-2144-6

ISSN : 0335-5985

Référence électronique

Samadia Sadouni, «Ahmed Deedat et l'islam indien en Afrique du Sud », Archives de sciences sociales des religions [En ligne], 139 | juillet - sepembre 2007, mis en ligne le 19 novembre 2010, consulté le 01 mai 2019. URL : http://journals.openedition.org/assr/9123; DOI : 10.4000/assr.9123 


\section{Samadia Sadouni}

\section{Ahmed Deedat et l'islam indien en Afrique du Sud ${ }^{1}$ Individualisme et transnationalisme islamique}

L'analyse actuelle des mouvements islamiques contemporains reflète un intérêt accru pour les nouvelles formes d'identités et de mobilisations transnationales des individus musulmans (Fourchard, Mary, Orayek, 2005 ; Hoeber Rudolph, Piscatori, 1997). De « nouveaux prédicateurs » autoproclamés (Haenni, Holtrop, 2002) font leur apparition dans le champ religieux et sur la scène médiatique où les dynamiques de l'individuation jouent un rôle central. Cependant, ces entrepreneurs religieux ne constituent pas un phénomène nouveau. C'est ce que montre l'exemple du prédicateur musulman sud-africain ${ }^{2}$, Ahmed Deedat ${ }^{3}$, qui participe à l'analyse des relations entre individualisation et globalisation : action individuelle sur la scène mondiale, d'un côté, rôle joué par la communauté transnationale (Kastoryano, $2000: 353)^{4}$ et l'État dans la construction des réseaux islamiques, indispensables à l'internationalisation de ses activités religieuses, de l'autre. C'est par le regard porté sur les relations entre l'individu, le groupe religieux et l'État que cet article poursuit l'étude du transnationalisme islamique.

Deedat a réactualisé, dans le contexte particulier de l'apartheid, un modèle de prédication islamique qui s'inscrit dans un registre aussi inédit que celui de la polémique islamo-chrétienne. L'engagement pour la défense de la cause islamique

1. Cet article reprend ma thèse en science politique : Sadouni (2004).

2. Les musulmans sud-africains représentent une minorité de moins de $2 \%$ de la population totale. L'islam d'Afrique du Sud est composé de quatre grands groupes de populations : la communauté métisse des Malais du Cap et les musulmans originaires du sous-continent indien qui constituent la très grande majorité des musulmans sud-africains, puis les Noirs, et enfin les musulmans originaires d'Europe.

3. Issu de la communauté indienne musulmane de Durban, Deedat a fait l'objet de rares études. Cependant, nous pouvons citer Riaz Cassim Jamal, (1991) ; nos précédents travaux : Sadouni (1997, 1999, 2000) et David Westerlund (2003).

4. «La communauté transnationale renvoie à des communautés composées d'individus ou de groupes établis au sein de différentes sociétés nationales, qui agissent à partir des intérêts et des références communs (territoriaux, religieux, linguistiques), et qui s'appuient sur des réseaux transnationaux pour renforcer leur solidarité par-delà les frontières nationales ». 
(Tayob, 1995) ${ }^{5}$ s'est construit en réaction au prosélytisme des missionnaires en Afrique du Sud et ailleurs. Ce nouveau type de discours religieux ne s'apparente ni au néo-fondamentalisme, ni à l'islamisme. Il s'agit plutôt d'une forme de communautarisme ${ }^{6}$ religieux qui ne s'entend pas ici comme isolement : elle reste liée par la rencontre de l' "Autre ». Dans l'espace transnational et dès les années 1970, la diffusion par l'usage des technologies modernes d'un discours rétablissant des frontières imaginaires entre musulmans et non musulmans, a visé l'universalisation d'un mode de prédication islamique qui crée du sens. C'est à partir de cette projection sur la scène mondiale qu'il faut voir comment l'individu construit son autonomie dans un espace communautaire fortement empreint des lois de ségrégation raciale résultant de l'apartheid, puis comment la communauté transnationale contribue à l'individualisation et aux stratégies internationalistes du prédicateur sud-africain.

En tant que figure médiatique de l'islam contemporain ${ }^{7}$, l'étude du parcours de Deedat a l'avantage de rendre compte de la complexité du champ religieux et des spécificités des pratiques religieuses dans la minorité musulmane d'Afrique du Sud. Cependant, l'intérêt porté à cet acteur de la mondialisation repose plus particulièrement sur l'étude des répertoires d'action dans un contexte autoritaire et colonial (Mamdani, 2004) ${ }^{8}$, celui de l'État d'apartheid marqué par l'activisme des missionnaires chrétiens. L'analyse socio-historique de sa carrière de prédicateur dégagera les prémisses contextuelles de son discours et de son action religieuse.

5. Le parcours de Deedat représente une des formes de la dynamique de réislamisation en Afrique du Sud. D'autres organisations au sein de la communauté musulmane sud-africaine ont participé au phénomène de résurgence de l'islam comme le Muslim Youth Movement influencé, entre autres, par l'islam politique (Tayob, 1995).

6. Le communautarisme, en tant que processus de reconstruction de structures collectives de type identitaire, ne désigne pas dans ce cas précis un enfermement communautaire que Deedat a, par ailleurs, fortement critiqué. Dans son discours de réislamisation au centre duquel se trouve la da'wa (appel à l'islam), le rapport à la religion n'isole pas le musulman de son environnement social même si le contexte de l'apartheid oblige les groupes ethniques à vivre dans un espace différent et séparé. En revanche, le néo-fondamentalisme selon Olivier Roy " s'adresse à l'individu qui décide explicitement de mettre sa vie sous le signe exclusif du religieux, et qui pour cela rompt avec l'environnement majoritaire» (Roy, 2005 : 131).

7. Deedat a reçu en Arabie Saoudite, le prix King Faisal International Award For Service to Islam, en 1986. Il était le premier sur le continent africain à recevoir ce prix international qui avait déjà récompensé des personnalités comme Maulana Abul 'Ala Maududi et Maulana Abul Hassan Ali Nadwi.

8. Mahmood Mamdani considère que « l'apartheid doit être appréhendé comme une forme d'État ». Il rappelle également que la nature du pouvoir et la forme de gouvernement déterminent la forme de révolte qu'elle suscite. 


\section{L'exemple du prédicateur-controversiste ${ }^{9}$}

Ahmed Deedat, né en 1918 en Inde, dans la province de Surat, décède le 18 août 2005 à son domicile familial situé à Verulam ${ }^{10}$, après avoir souffert pendant neuf ans d'une paralysie générale. Il avait quitté l'Inde, en 1927, pour rejoindre son père, tailleur de profession, installé à Durban. Son origine sociale et son parcours migratoire influenceront fortement son système de représentation et sa pensée. La communauté indienne musulmane dont il est issu est essentiellement dirigée par une élite commerciale gujaratie qui a reproduit en Afrique du Sud les mêmes formes de communautarisme qu'en Inde. La stratification communautaire, héritée du système des castes, était telle qu'un fils de tailleur avait peu de chances de s'imposer dans le champ religieux dominé par les lettrés en islam et les riches marchands. Faut-il voir dans sa trajectoire une volonté de revanche de " parvenus ", jusque-là exclus des positions de pouvoir par les héritiers d'une "bourgeoisie de communauté » ? Le contexte doublement minoritaire et colonial sera déterminant dans la construction et le succès, au sein de sa communauté d'origine, d'un tel discours islamique. Autodidacte ayant interrompu ses études à l'âge de 16 ans, il n'est pas un 'alim (savant musulman) et n'a reçu aucun enseignement universitaire, pas même dans l'étude de l'islam.

L'expérience sud-africaine, marquée par l'idéologie politique de l'apartheid et la «montée du prosélytisme » (Clémentin-Ojha, Gaborieau, 1994) en Afrique, participe à la construction d'un modèle original de polémique religieuse. Deedat se lance dans l'étude comparée des religions afin de protéger les musulmans du prosélytisme des missionnaires, en particulier de celui des Témoins de Jéhovah et des born again Christians (Deedat, s.d. : 62) ${ }^{11}$. Ses connaissances théologiques, acquises sur un mode de " bricolage » dans le champ islamique mais aussi chrétien, ont renforcé son autonomie d'acteur religieux. Elles ont alimenté sa réflexion pour donner un caractère original à sa prédication. Le modèle du chrétien évangélique, acteur incontournable du continent africain, a représenté une source d'inspiration dans l'édification de son mode original de da'wa. Il en a repris les stratégies de prosélytisme: la distribution à grande échelle de ses opuscules ${ }^{12}$ ainsi que l'usage de la vidéo. En Afrique du Sud, ses débats avec des pasteurs pentecôtistes et de la NGK (Nederduitse Gereformeerde Kerk), la principale Église calviniste réformée des Afrikaners, ainsi que la controverse avec le baptiste

\section{Mon expression.}

10. Verulam se trouve à 25 kilomètres au nord de Durban, centre portuaire et industriel, et troisième ville la plus importante de la république sud-africaine.

11. L'événement qui marque sa vocation de prédicateur est sa rencontre en 1939, à l'âge de 21 ans, avec les missionnaires et étudiants chrétiens du séminaire, Adams Mission College, près de Durban. Leur prosélytisme a été vécu comme une agression.

12. Le réseau de distribution des ouvrages de Deedat, traduits en plusieurs langues, servait une stratégie internationale caractéristique des mouvements modernes de propagation religieuse. Toutefois, ces moyens ne suffisaient pas à satisfaire son ambition car il indiquait : "Si nous en avions les moyens, nous inonderions le monde de notre littérature ». 
John Gilchrist ont façonné un nouveau champ d'action religieux délaissé par les «clercs-savants ${ }^{13}$. La trajectoire de Deedat représente, en effet, une des formes de réislamisation liées à la crise de l'autorité et au procès d'individualisation de la fonction religieuse en Afrique du Sud. Toutefois, sa dynamique d'individualisation du rapport au religieux s'est construite avant tout en réaction au prosélytisme chrétien qu'il a vécu comme une humiliation dès son vingt et unième anniversaire (Lubbe, 1987) ${ }^{14}$. La contestation de l'autorité traditionnelle des oulémas n'est que le prolongement de la frustration d'un entrepreneur religieux s'érigeant en défenseur des musulmans et, de manière plus générale, de l’islam.

C'est la découverte de l'ouvrage de Maulana Rahmat Allah Kairanawi, Izhar al-haq (manifestation de la vérité), qui lui donnera les outils indispensables à l'étude comparée des textes sacrés dans le cadre de ce débat polémique. La réappropriation du discours de Kairanawi, et plus précisément du modèle indien des munâzara-s (débats) du XIX ${ }^{\mathrm{e}}$ siècle, représente un événement fondateur dans sa carrière de prédicateur. Kairanawi, un savant musulman né à Kiranah, dans la banlieue de New Delhi, en 1817, avait exposé les principes d'argumentation à faire valoir face au missionnaire chrétien, le révérend Carl Pfander qui opposait la Bible comme source de la révélation au Coran, dans un livre traduit en arabe, Mizan al-haq (Walls, 1999). Après son séjour au Caucase, Pfander devait rejoindre en 1840 la Church Missionary Society en Inde où il efforça de mener sa mission de prosélytisme parmi les musulmans. Sa confrontation avec Kairanawi, notamment lors d'un débat public à Agra en 1854 et en présence de représentants du pouvoir colonial, a marqué l'histoire de la polémique religieuse entre chrétiens et musulmans en Inde avant la révolte des Cipayes de 1857 (Powell 1993). Leurs publications controversées et traduites dans plusieurs langues, ont alimenté les discours des futurs prédicateurs soucieux de faire triompher la "vérité » de la Bible ou du Coran.

Deedat a souvent reconnu s'être inspiré des méthodes employées par Kairanawi pour défendre l'islam du prosélytisme chrétien qu'il considère comme agressif et d'origine occidentale. Seule la controverse religieuse est susceptible, selon lui, de protéger les musulmans insouciants du danger qui les menace malgré la « croisade » qui se poursuit. Convaincu que ses activités permettent d'agir au mieux dans le cadre de la da'wa et d'interagir avec les missionnaires chrétiens, il encourage d'autres musulmans à prendre la relève et à améliorer ses méthodes de prédication. La religion est pour lui plus qu'un ensemble de dogmes et de rites : il considère que le musulman a une mission à remplir en dépit du contexte dans

13. Dès les années 1960, il débattra en Afrique du Sud avec l'évangéliste américain Cyril Simkins, professeur de philosophie, de psychologie et d'exégèse du Nouveau Testament à la Johnson Bible College, sous le thème "le Christ a-t-il été crucifié ? "Sur la scène mondiale, il débattra notamment avec le télévangéliste américain Jimmy Swaggart, le Palestinien chrétien Anis Shorrosh, le pentecôtiste suédois Stanley Sjöberg.

14. D'autres événements ont par la suite renforcé sa position de polémiste avec, notamment, les propos tenus par l'Église anglicane et la NGK contre l'islam dans les années 1960 et 1980. 
lequel il vit. Il encourage la communauté musulmane à s'ouvrir sur son environnement afin de transmettre le message islamique. Le prosélytisme religieux et l'apprentissage des systèmes de croyances et des représentations collectives de l' "Autre » deviennent un mode d'action du revivalisme islamique qu'il considère comme indispensable. Lors d'une conférence dans les Émirats Arabes Unis, il avait ainsi conseillé aux Arabes d'acquérir la traduction anglaise du Coran afin de prêcher aux non musulmans qu'ils rencontraient. Ainsi la langue arabe, qu'il n'utilise que pour la récitation du Coran, n'est plus considérée comme le medium indispensable à la prédication islamique : l'anglais reste le véhicule d'un discours religieux qui vise l'Occident ${ }^{15}$. À partir de ses propos, son système de représentation et sa vision globale du monde peuvent se résumer ainsi : " mon humble opinion est qu'il y a deux genres de personnes dans ce monde, les musulmans et les non musulmans ${ }^{16}$.

Or, on peut s'interroger sur le caractère innovant de son mode de prédication et sur l'impact de l'internationalisation de ses activités sur son discours. L'analyse de sa dernière conférence, qui a eu lieu à Sydney en avril 1996 et a clos sa carrière de prédicateur international, permettra d'y répondre.

\section{Innovation ou réinvention de la polémique religieuse ?}

L'adoption de stratégies strictement internationalistes a toujours constitué un objectif pour Ahmed Deedat sans pour autant modifier la nature de son discours ni même sa représentation du monde. Sa «mission » de prédicateur international implique, cependant, un désengagement progressif de la scène locale. C'est ce que montre l'exemple de l'islamisation des populations noires en Afrique du Sud. En 1961, il décide de prendre en charge la formation de "missionnaires" musulmans à As-Salaam, une zone rurale située à soixante kilomètres au sud-ouest de Durban, où il construit la première mosquée. Après y avoir consacré douze ans, il propose au Muslim Youth Movement de prendre la direction d'As-Salaam afin de le libérer de la charge d'islamisation qui représente ici la frontière entre le local et le global. Fort de ses talents de débatteur et de conférencier, il reste persuadé que ses efforts doivent se concentrer sur la scène médiatique et mondiale, ce qu'illustre, entre autres, sa dernière conférence organisée à Sydney, au Brisbane City Hall, une salle pouvant accueillir 35000 personnes et où s'était déjà rendu le célèbre télévangéliste américain, Billy Graham.

En costume et coiffé d'une calotte musulmane blanche, Deedat se distingue des oulémas lors de ses conférences et débats. Le port de la barbe reste bien entendu le symbole du respect de la sunna mais le costume occidental renseigne

15. Deedat n'utilisait que l'anglais dans ses conférences à l'étranger, y compris celles organisées dans les pays du Golfe. et s'adressait uniquement en anglais à ses interlocuteurs arabes.

16. Communication présentée lors de la quatrième convention annuelle du Muslim Youth Movement en Afrique du Sud. 
sur sa volonté de se différencier du «clerc-savant». Deedat ne lit pas de notes, seulement des citations de documents (articles de journaux, passages de livres) pour illustrer son argumentation qui reprend les principales thématiques de ses ouvrages et opuscules. Son discours, basé sur une collection de savoirs éclatés, repose autant sur un corpus religieux, composé de citations puisées dans le Coran et la Bible, que profane.

Dans le cadre de cette conférence fortement critiquée par des leaders chrétiens du pays, il utilise des méthodes de provocation, déjà éprouvés à Durban. Il organise l'événement pendant la fête des Pâques et plus précisément le jour de la passion du Christ. Il s'adresse à son public sur un ton assuré qui renforce l'exhortation et insiste, entre autres, sur le fait que le Coran ne fait que confirmer la prophétie de Jésus. Il conclut en faisant un geste de la main qui exprime sa désapprobation à l'égard des chrétiens : "And you think that we are the ennemies ».

Il invite les dignitaires religieux d'Australie à un débat mais demande que soit organisé un symposium qui reposerait essentiellement sur l'exposé des arguments de chacun des orateurs face auquel le public ferait son choix. Le choix est ici une rhétorique constante chez le prédicateur sud-africain qui, sur la forme, reprend à son compte les techniques de prosélytisme des débatteurs, Pfander et Kairanawi, au XIX ${ }^{\mathrm{e}}$ siècle en Inde. Sur le fond, son dernier ouvrage, The Choice, qui représente une compilation de l'ensemble de ses écrits, illustre sa stratégie de prosélytisme : se recentrer sur l'individu. Ce qui implique un travail d'argumentation et d'interprétation du texte religieux pour convaincre l' «Autre » de la finalité du message de l'Islam.

Fort d'un « don personnel » d'orateur, il sait captiver son auditoire, tant par son attitude et le ton de sa voix que par le choix de ses formules. Le travail des cadreurs, les gros plans sur Deedat participent à la mise en scène héroïque de son action et renforcent l'image de "champion de la $d a^{c} w a »$ que lui reconnaît un journal musulman du $\mathrm{Cap}^{17}$. De la même manière que le camp meeting organisé par les télévangélistes, ses conférences et débats constituent pour d'autres « un habile mélange de religion et de divertissement » (Gutwirth, 1998 : 12). Cependant, son succès au sein de sa communauté d'origine d'abord, puis dans différents pays du monde musulman (États arabes, Afrique sub-saharienne, Pakistan, Malaisie) et d'Occident (États-Unis, Angleterre, France, Australie), s'explique avant tout par l'apologie de l'islam et la fierté d'être musulman privilégiées dans son discours. L' " estime de soi », devenant ainsi un enjeu collectif (Ion, 2001), aboutit à la mobilisation d'une communauté religieuse qui cherche à se définir contre des modèles culturels concurrents et dominants.

L'internationalisation de ses activités religieuses a donc représenté une innovation plus qu'une réinvention du modèle des munâzara-s. Pendant près de quarante

17. «The legacy of Shaikh Ahmed Deedat », Muslim Views, août 2000, p. 2. 
ans, son discours religieux et son style d'orateur n'ont pas varié. Deedat va du local au global en reprenant les thèmes des débats et les arguments avancés depuis des générations : la trinité, les corruptions dans la Bible, la crucifixion du Christ. Deedat ne fait que reprendre ces sujets selon une approche personnelle qui répond aux attentes d'un public musulman soucieux de défendre son identité religieuse qu'il estime malmenée par les médias occidentaux.

\section{Un nouveau mode d'action transnationale}

Ces «formes inédites de transnationalités religieuses" (Colonomos, 2000 : 14) s'inscrivent, on l'a vu, dans le cadre des rapports de puissance entre l'islam et le christianisme en Afrique essentiellement. Elles empruntent les unes aux autres tout en s'adaptant aux environnements sociaux spécifiques dans lesquels elles opèrent. Dans les années 1980, Deedat avait un objectif : celui de faire face à la nouvelle version du prosélytisme protestant d'origine américaine en utilisant à son tour des techniques modernes de communication pour en faire des instruments de défense de l'islam ${ }^{18}$. Aussi, son succès médiatique sur les chaînes de télévision arabe au milieu des années 1980, dans les librairies islamiques, sur les sites Internet, s'inscrit dans le phénomène des "idéoscapes » qu'Arjun Appadurai considère comme les nouveaux instruments identitaires de la communauté en construction (Appadurai, $2001: 33$ ).

Le recours aux technologies modernes de communication et la modernisation de son organisation Islamic Propagation Centre International (IPCI) ${ }^{19}$, servent la stratégie internationaliste du prédicateur sud-africain au même titre que celle des télévangélistes. C'est la transmission sur la scène internationale de son modèle de polémique religieuse qui lui octroie du pouvoir et un statut d'acteur dans la mondialisation de l'islam (Asad, 1993 : 13) ${ }^{20}$. La dimension charismatique du personnage s'est renforcée avec la production accélérée et la distribution à grande échelle de ses pamphlets, opuscules, cassettes vidéo, qui sont les produits dérivés de ses conférences et débats. La commercialisation de ses vidéos a remporté un succès international important, notamment celle du débat avec le télévangéliste

18. Lors d'un interview donné à une journaliste d'une chaîne de télévision australienne, Deedat affirme qu'il existe en Afrique 35000 «crusaders » venus d'Amérique du Nord et 6000 en Indonésie. Vidéo de la conférence de Deedat, « Easter- A Muslim View Point », 5 avril 1996.

19. L'IPC, créé en 1957 par Ahmed Deedat et Goolam Hoosen Vanker, devient en 1986 une organisation internationale, l'Islamic Propagation Centre International, dont Deedat fut président de 1958 à 1999. La personnalisation de l'organisation se traduit par la promotion de Deedat avec notamment la production et la distribution à l'étranger de ses vidéos et ouvrages. Toutefois, l'IPCI remplissait d'autres fonctions de la da'wa comme la publication de corans traduits en anglais, l'éducation islamique des nouveaux convertis, la formation internationale de prédicateurs africains.

20. Talal Asad souligne le transfert de pouvoir dans les religions monothéistes : " when a project is translated from one site to another, from one agent to another, versions of power are produced ». 
Jimmy Swaggart en 1986. Des musulmans de différentes parties du monde se sont identifiés à l'image mythique et valorisante qu'il propose de l'islam. C'est ainsi que l'on peut distinguer chez ce prédicateur une forme d'autorité renforcée par son parcours de prédicateur itinérant, comparable à celle de Billy Graham dont il admirait le charisme. En outre, cette construction identitaire évoque singulièrement des modes de mobilisation des télévangélistes américains. Il a, en effet, procédé dans la construction médiatique de son discours à des emprunts au télévangélisme sans perdre pour autant ses particularismes, ni ses originalités. Dès le début de sa carrière de prédicateur-controversiste dans les années 1950, il avait déjà à sa disposition différents stocks idéologiques bricolés pour façonner son système de représentation et son modèle de la polémique religieuse.

Le rappel de l'antériorité du modèle indien de polémique islamo-chrétienne ainsi que l'analyse des formes de mimétisme participent bien à la compréhension de ce nouveau mode de militantisme musulman que Deedat a réussi à ériger comme mode de réislamisation en Afrique du Sud et ailleurs. Ce modèle est loin d'être obsolète. D'autres après lui ont su le reproduire en innovant à leur tour. C'est le cas de l'Indien Zakir Naik, basé à Bombay, auquel Deedat reconnaissait des qualités d'orateur en le qualifiant de «Deedat + ». La polémique religieuse, désormais déconnectée du contexte social et politique, s'inscrit dans un processus de construction identitaire déterritorialisée (Robertson, 1993).

\section{Communauté, État et réseaux}

La diffusion sur la scène mondiale des activités religieuses de Deedat s'est réalisée avec le soutien conjoint de trois acteurs : la communauté musulmane locale, la communauté musulmane transnationale et l'État saoudien, essentiellement. Dès le début des années 1950, sa communauté d'origine, en Afrique du Sud, lui a permis de diffuser sur la scène locale son discours puis, dans les années 1970, lui a offert l'opportunité de s'intégrer dans un réseau transnational de solidarité islamique au centre duquel se trouve l'Arabie Saoudite. Fort de sa notoriété dans les pays du Golfe, le prédicateur sud-africain a, par la suite, réussi à construire son propre réseau avec le soutien financier de mécènes arabes, essentiellement des hommes d'affaires ${ }^{21}$. Ainsi, la construction de l'individu sur la scène mondiale ne s'est pas faite en dehors de la communauté même lorsqu'il a réussi à construire son propre réseau essentiel à son entreprise religieuse.

La communauté et l'État ont eu un impact décisif sur le déclenchement de l'action religieuse du prédicateur sud-africain. La formulation de sa subjectivité et de sa particularité est d'abord une réalité collective que représente ici la communauté locale, c'est-à-dire la communauté indienne dont l'État ségrégationniste a contribué

21. Deedat sollicitait souvent son appareil partisan transnational constitué de personnalités prêtes à financer ses activités religieuses. 
à la structuration en un espace religieux. Deedat a mené ses activités de prédication librement, sans redouter de censure gouvernementale, grâce à son mode d'action communautariste. L'apartheid, ayant pour fondement la liberté accordée à chaque groupe ethnique de disposer de son territoire ${ }^{22}$, a renforcé et encouragé ce type de communautarisme indien musulman. L'organisation de la religion ne concernait pas l'État mais la communauté. L'autonomie accordée à la minorité musulmane dans la gestion des affaires religieuses représentait pour ses principaux partisans la possibilité de vivre leur religion sans craindre une déculturation au contact de l'" Autre».

Néanmoins, le politique n'est pas absent de sa démarche militante pour la défense des musulmans qui repose, aussi bien en Afrique du Sud que sur la scène mondiale, sur la confrontation et la provocation religieuse. Dans le conflit avec la ville de Durban au sujet des signes religieux, Deedat a retourné à son avantage le système de ségrégation ethnique pour mener ses activités prosélytes. À la suite de nombreuses protestations relayées par des pasteurs, l'IPCI se voit tout d'abord interdire l'érection de panneaux appelant à l'islam dont un signe lumineux « Al Qu'ran, the Last Testament». Invoquant le droit des Indiens musulmans de disposer librement de leur zone séparée, l'organisation de Deedat a gain de cause et, par la même occasion, oppose l'hostilité des Blancs chrétiens à la tolérance des Noirs chrétiens. Cet épisode ne constitue par pour autant un changement dans sa représentation religieuse de l'apartheid. Deedat considère en effet que seul «le christianisme en est responsable et que l'islam a, en revanche, toutes les réponses »(Esack, 1997 : 218). Il établit une relation entre le christianisme, en tant que religion, et la nature raciste de l'État sans prendre en compte l'idéologie du «peuple élu » qui n'est pas partagée par toutes les Églises. Certaines d'entre elles ont, au contraire, participé à la lutte contre l'apartheid mais le schéma cognitif de Deedat est un prisme qui sélectionne les informations pour illustrer l'argumentation.

Les ressorts politiques ambivalents de la polémique religieuse se traduisent par l'expression du mécontentement communautaire en même temps que par un désengagement du champ politique. Ce mode de contournement de l'autoritarisme du régime explique le soutien financier de riches marchands aux activités de Deedat correspondant à une division du travail religieux ${ }^{23}$. Il remplit pour les leaders de sa communauté une fonction dans la défense de la dignité des musulmans face au racisme d'un pouvoir blanc et désigné comme chrétien. C’est

22. Le régime de Pretoria reconnaissait quatre groupes raciaux : les Blancs, les Coloureds, les Indiens et les Noirs, dotés de droits différents. Des législations discriminatoires en matière d'espace, de travail, de formation, de logement, de revenus et de contrôle des déplacements ont consolidé la politique de développement séparé.

23. Le mouvement de réislamisation en Afrique du Sud est caractérisé par une pluralité d'organisations qui remplissent différentes fonctions dans l'espace communautaire : l'éducation, l'aide humanitaire, la $d a^{6} w a$, etc. 
dans ses relations communautaires qu'il trouve au début des années 1950 ses principales ressources politiques, matérielles et financières.

En Afrique du Sud, aucun 'alim (savant, singulier de oulémas) n'avait abordé l'étude comparative des religions ou la prophétie de Muhammad comme «la plus logique des prophéties de la Bible ». Déjà, sa première conférence publique en 1955 à Durban portait sur « Muhammad dans l'Ancien et le Nouveau Testament ». Le fil directeur de cette première conférence publique visait à démontrer aux chrétiens que l'achèvement de leur religion était dans l'islam, dernière religion monothéiste. En reprenant des arguments traditionnels contre un christianisme qui aurait occulté l'annonce du prophète Muhammad et à travers lui l'islam, Deedat contribue, à la fois, à la négation et à la réaffirmation des règles de la société (Bennani-Chraïbi, Fillieule, 2003 : 64-66). Le sentiment d'insécurité et le ton alarmiste qu'il entretient dans son discours de défense des musulmans participent finalement au renforcement des frontières entre les différents groupes ethniques dans l'espace national, et plus particulièrement entre "Indiens » et "Blancs ».

La situation se renverse, dans les années 1980, avec l'émergence d'une nouvelle génération de militants musulmans. Ceux-ci se montrent très critiques vis-à-vis d'un confessionnalisme exacerbé qui se fait au dépend de la lutte anti-apartheid à laquelle ils se joignent aux côtés des chrétiens ${ }^{24}$. Toutefois, l'évènement qui marque une rupture dans le soutien communautaire est, en 1995, la promotion de la vidéo sur l'hindouisme, intitulée "Concept of God in Hinduism and Islam ». Même si les musulmans d'Afrique du Sud reconnaissent son rôle essentiel dans la valorisation de l'islam, ils lui reprochent l'usage de procédés insultants et agressifs dans la description des textes sacrés qu'elle tourne en dérision. Un conflit avec la communauté hindoue en résulte, qui se traduit notamment par des menaces de boycott des commerces musulmans et par une campagne de dénigrement de l'islam ${ }^{25}$. Trente et une associations islamiques, dont des membres d'organisations de oulémas, décident de se réunir lors d'une conférence publique intitulée, "The Quranic Prophetic Method of Propragating Islam », pour proposer un discours alternatif à celui de Deedat, que, pour autant, ils ne dénoncent pas publiquement ${ }^{26}$. Les nombreuses pressions communautaires n'ont cependant pas raison de sa détermination à poursuivre sa controverse religieuse et ce n'est qu'en 1999 que les nouveaux membres du conseil d'administration de l'IPCI acceptent de retirer de la vente et de la distribution la vidéo sur l'hindouisme. À travers cet

24. Le dialogue interreligieux s'est, en effet, invité dans le champ politique symbolisé par le front anti-apartheid United Democratic Front réunissant entre autres le Natal Indian Congress, des associations liées à l'African National Congress (ANC), des organisations syndicales, des Églises, des clubs sportifs. En outre, la création d'une branche sud-africaine de la World Conference on Religion and Peace a également participé au dialogue des religions.

25. Ces menaces ont été essentiellement relayées par le South African Hindu Maha Sabha et le National Hindu Front créé à la suite de la sortie de la vidéo de Deedat en 1995.

26. M. MaKKI, The Muslim Digest, 46, no 6 et 7, janvier-février 1996, pp. 289 sq. Jaspreet KInDRA, " Pressure mounts against Deedat », The Leader, 15 décembre 1995. 
exemple, l'impact de Deedat est à nuancer au sein de sa communauté d'origine qui comprend combien ses activités de prosélytisme ne répondent plus à l'attente des musulmans aux lendemains des premières élections démocratiques dans le pays. L'absence d'appareil partisan a fortement limité sa capacité à trouver des relais dans la propagation de son modèle de da'wa dans l'espace stato-national depuis les années 1980. Il reste avant tout un concepteur moderne de la polémique islamo-chrétienne dont certains continuent à admirer le charisme. C'est en Arabie Saoudite, essentiellement, que Deedat trouvera ses principaux mécènes et soutiens dans la médiatisation de ses activités.

\section{Communauté transnationale et déterritorialisation}

Les relations conflictuelles et les rivalités internes de sa communauté d'origine ont amené Deedat à se tourner vers une communauté transnationale qui devait lui permettre de poursuivre ses activités et de diffuser son discours sur la scène nationale et mondiale. Sa projection sur la scène internationale repose avant tout sur une ambition et une stratégie personnelles de l'acteur religieux, mais elle s'accompagne progressivement d'identifications à l'hypercommunauté gravitant autour de l'État saoudien ${ }^{27}$. Cette hypercommunauté s'organise sous forme d'un réseau transnational et s'apparente à une solidarité organique internationale qui ne peut se construire sans individualités et sans État. L'État, ici, n'est pas l'État d'origine d'un groupe immigré, mais un État souhaitant participer au financement d'un réseau d'associations de croyants issus de différents contextes culturels et migratoires. Il y a, dans le cadre de ces interactions entre politique et religieux, échange d'utilités et intérêts communs à coopérer dans un but ultime : la valorisation de l'islam face à un Occident considéré comme hégémonique.

La communauté transnationale représente ici une alternative à la communauté classique, une nouvelle forme de communalisation. Elle n'est pas nouvelle en soi mais s'est développée avec l'avènement des nouvelles technologies de communication modernes (Allievi, 2003). La minorité musulmane sud-africaine s'est trouvée, très tôt, travaillée par la déterritorialisation, sous l'effet de la circulation des idées et des migrations. Le parcours international de Deedat s'explique en partie par l'impact de la longue tradition du transnationalisme dans l'islam indien. Afin d'atténuer le caractère minoritaire de l'islam local en Afrique du Sud, les acteurs indiens de la première vague de réislamisation du début $\mathrm{du} \mathrm{XX}^{\mathrm{e}}$ siècle se sont fortement inspirés des activités islamiques des leaders religieux du sous-continent indien comme Maulana Abd Aleem Siddiqui. Ce dernier avait inauguré une trajectoire de prédicateur internationaliste en donnant des conférences dans

27. Deedat ne s'est pas orienté par défaut vers une transnationalisation de ses activités religieuses après avoir été confronté, dans son pays, à un affaiblissement du soutien communautaire. Son parcours s'inscrit, comme nous allons le voir, dans le processus de transnationalisation islamique qui représente un nouvel espace de la réislamisation. 
différents pays et en débattant de l'islam avec le célèbre écrivain, et prix Nobel, Georges Bernard Shaw. Dès les années 1930, on retrouve des figures internationales de l'islam indien dont le parcours s'inscrit dans ce qu'on appelle aujourd'hui la mondialisation.

Toutefois, c'est au cours des années 1970 que les acteurs sud-africains de la réislamisation se sont tournés vers le monde arabe et les États-Unis. Ce transnationalisme islamique n'est pas le produit d'une mobilité d'immigrants entre leur pays d'origine et leur pays d'accueil ${ }^{28}$. Il s'agit d'un réseau de solidarité islamique composé d'acteurs issus de différents espaces nationaux (États-Unis, Angleterre, Afrique du Sud, Arabie Saoudite, Pakistan, Malaisie) et d'origine ethnique différente (Américains d'origine arabe, Afro-américains, Sud-africains d'origine indienne, Européens convertis à l'islam, etc.) ${ }^{29}$.

L'intégration au sein de réseaux transnationaux dominés par la Muslim Students' Association des États-Unis et du Canada et par la World Assembly of Muslim Youth (WAMY) constitue une première étape dans l'internationalisation des activités de Deedat. La WAMY, fondée en 1972 et basée à Riyad, lui a procuré des ressources financières au début de sa carrière mondiale en 1976. Le plus souvent, cette aide était conditionnée à sa participation aux rencontres internationales organisées en Afrique ou en Asie du Sud-Est. La transnationalisation de ses activités s'est ainsi effectuée par un séjour saoudien et par des contacts étroits avec les organisations islamiques d'Amérique du Nord. Ces mêmes connexions, s'effectuant selon une triangulaire entre l'Afrique du Sud, les États-Unis et les pays arabes, lui ont permis d'assurer un rôle d'acteur international. Ce réseau de réislamisation fait de relations individuelles, d'échanges et d'utilités, s'articule autour d'organisations et de personnalités liées par un intérêt identitaire commun. Cet espace auquel appartient Deedat n'est ni global, ni local, il s'est constitué à partir de la libre association de croyants soucieux de construire un réseau transnational de réislamisation.

Par le biais de ses institutions internationales, l'État saoudien occupe une place importante dans la formation de ces espaces transfrontaliers. Son rôle essentiel dans la recomposition des identités transnationales, aptes à transcender les clivages ethniques, nationaux et linguistiques, s'exerce par de nouvelles formes de patronage pour la promotion d'une identification commune : l'appartenance

28. Différents travaux portent sur les communautés transnationales européennes qui relient le pays d'origine au pays de résidence. Voir notamment, Valérie Amiraux (2001) ; Riva Kastoryano (1997).

29. La trajectoire transnationale de Deedat n'est pas ancrée dans son pays d'origine, qui représente essentiellement une source d'inspiration dans la construction de son modèle de la polémique islamo-chrétienne. En outre, le lancement de sa vidéo controversée sur l'hindouisme confirme sa volonté de distanciation à l'égard de l'Inde qui, marquée par le nationalisme hindou, le lui rend bien en rejetant sa demande de visa en 1995. 
à l'islam (Sheikh, $2003: 56)^{30}$. Deedat avait compris que le soutien de l'Arabie Saoudite était indispensable à son parcours de prédicateur-controversiste et à l'internationalisation de ses activités. Les réseaux islamiques transnationaux qu'il avait intégrés et caractérisés par des «liens faibles» (Degenne, Forsé, 1994 ; Colonomos, 1995) lui ont facilité l'accès aux différentes institutions islamiques internationales. En contrepartie de cette relation privilégiée avec la WAMY, la Rabita, Deedat a soutenu le royaume saoudien dans sa politique internationale, par exemple lors de la guerre du Golfe en 1991. Dans ce cas précis, la mobilisation n'est pas uniquement celle des ressources mais aussi celle des loyautés (Chazel, 1993 : 159). Le prédicateur sud-africain a rempli différentes fonctions identitaires et politiques qui caractérisent la multiplexité du réseau auquel il appartient.

\section{Personnalisation d'un réseau utilitaire}

Aux débuts de sa carrière, Deedat et ses proches collaborateurs issus essentiellement du Muslim Youth Movement en Afrique du Sud ont cherché à exporter son discours religieux sur le marché islamique mondial. Il suffisait de trouver des agents financiers prêts à investir dans l'internationalisation de ses activités par le financement de l'Islamic Propagation Centre International (IPCI) ${ }^{31}$. La multi-position dans l'espace transnational lui a permis de construire son propre réseau de solidarité islamique indispensable à son ambition mondiale. Progressivement Deedat s'est distancé de l'ancrage communautaire transnational en construisant son propre réseau utilitaire, producteur de solidarités nouvelles. Souhaitant s'émanciper des ressources aléatoires de la WAMY, il trouve d'autres partenaires financiers dans les pays du Golfe qui forgeront sa réputation de prédicateur international. Ces principaux soutiens et mécènes, représentés par les hommes d'affaires et de pouvoir arabes, ont vu en lui un acteur apolitique essentiel à la valorisation de l'islam, capable de mieux concurrencer la domination d'un monde occidental qu'ils perçoivent comme chrétien et agressif à l'égard des musulmans. Cette vision antagoniste de l'Occident et de l'islam continue à alimenter le discours du fondamentalisme religieux aussi bien chez les chrétiens que chez les musulmans. L'organisation de son propre réseau s'explique par la rencontre d'un modèle polémique du religieux avec les importantes ressources financières de particuliers arabes. Elle a facilité son ascension en tant qu'acteur international. Selon Manuel Castells, "la performance d'un réseau donné va donc dépendre de deux de ses qualités essentielles : sa connexité, c'est-à-dire

30. L'Arabie Saoudite ne cherche pas dans sa politique de panislamisme l'hégémonie politique. Comme le souligne Naveed S. Sheikh, " the Saudi government was seeking to exercise selective leadership, without an identifiable overall responsability. It did not seek hegemony, but its sole purpose was to prevent others from achieving it ».

31. Il faut souligner par ailleurs que l'IPCI a des ressources financières bien inférieures à celles des organisations des télévangélistes américains. L'organisation de J. Swaggart représentait, par exemple, au milieu des années 1980 une vaste entreprise de 1500 salariés. Cf. Jacques Gutwirth, op. cit. 
sa capacité structurelle à faciliter la communication entre ses composants; sa cohérence, c'est-à-dire le degré de communauté d'intérêts entre les objectifs du réseau et ceux de ses composants "(Castells, 1998 : 207). Deedat est devenu un entrepreneur religieux indépendant dès lors qu'il a su contrôler ses propres ressources en sollicitant l'aide matérielle et financière de son appareil partisan dans les pays du Golfe. Il a su, grâce à un carnet d'adresses fourni, réunir des fonds importants pour transformer son organisation en une entreprise rentable. L'investissement dans l'achat de bâtiments commerciaux lui a procuré des revenus réguliers indispensables à la poursuite de ses activités de prédication mais aussi à son émancipation et à l'individualisation de son parcours transnational ${ }^{32}$.

Cette logique capitaliste qui sous-tend son action religieuse était déjà présente chez les premiers acteurs du mouvement de réislamisation sud-africain. Le capitalisme et la défense de l'identité islamique représentaient, par ailleurs, les principaux domaines investis par les riches marchands indiens dès le début du $\mathrm{Xx}^{\mathrm{e}}$ siècle $^{33}$. En tant qu' " entrepreneurs communautaires » et agents intermédiaires des pouvoirs publics, les commerçants musulmans ont considéré le capitalisme comme essentiel à l'intégration et l'ascension sociale des musulmans en Afrique du Sud et à l'organisation moderne de l'islam. On retrouve donc dans cette logique capitaliste deux niveaux de justification : " les justifications individuelles (en quoi une personne trouve des motifs à s'engager dans l'entreprise capitaliste) et les justifications générales (en quoi l'engagement dans l'entreprise capitaliste sert le bien commun) » (Boltanski, Chiapello, 1999 : 45). Deedat a cherché à obtenir des moyens financiers au sein de sa communauté d'origine, puis dans les pays du Golfe. Son ambition internationale, dépendante d'une manne financière importante, visait l'institutionnalisation de son discours en Afrique du Sud - ce qui obtint plus ou moins de succès - et sa diffusion à l'échelle mondiale. Sa trajectoire personnelle s'est renforcée avec le soutien financier obtenu des mécènes arabes qui lui reconnaissent des qualités d'orateur dans son action contre le prosélytisme des évangélistes en Afrique et ailleurs.

L'IPCI sert, encore aujourd'hui, d'instrument efficace dans la régulation et la circulation des produits et de la pensée du prédicateur ${ }^{34}$. En outre, sa rencontre

32. D'après la définition de l'organisation, donnée par Manuel Castells, l'IPCI représente ce «système de moyens structuré autour de l'intention de réaliser des objectifs spécifiques ", Castells, p. 207.

33. Ces derniers ont non seulement protégé leurs intérêts commerciaux en contestant les lois « anti-indiennes », par la création d'organisations politiques comme le Natal Indian Congress, mais ont été aussi les acteurs-clés de l'institutionnalisation de l'islam dans l'ancienne province du Natal. Leur mobilité sociale et politique a contribué à la mise en place de structures modernes d'autosuffisance en matière d'éducation et d'économie notamment.

34. Depuis l'attaque cardiaque de Deedat en 1996, les ressources financières de l'IPCI ont diminué. Mais, depuis son décès, l'organisation continue à promouvoir son discours en commercialisant ses DVD et à organiser des stages de formation dans l'étude comparative des religions (www.ahmed-deeedat.co.za). Cependant, les continuateurs de son projet et de son style de prédication médiatique sont des individus qui, à l'instar de l'Indien Z. Naik, se sont réappropriés son discours dans le contexte particulier de leur société nationale. 
avec différents chefs d'État et leaders musulmans, comme le président pakistanais, le général Zia-ul-Haq, le Soudanais Hassan Tourabi, le Cheikh Zayed bin Sultan al Nahyan des Émirats Arabes Unis, confirme l'ambition internationale de Deedat. Une ambition qui s'apparente à celle des télévangélistes.

\section{Conclusion}

Le modèle de la polémique islamo-chrétienne que Deedat a innovée dans le cadre de la globalisation rend compte de dynamiques d'individualisation qui n'excluent pas pour autant le pluralisme des références, la résistance à l'autorité, la mobilisation formelle des ressources et la politisation des identités par l'allégeance à un État.

Toutefois, il a fallu plus de trente ans à Deedat avant qu'il ne réussisse à médiatiser son discours religieux sur la scène mondiale. On constate qu'après son décès il reste très présent sur les sites Internet. Son succès médiatique s'explique essentiellement par le caractère identitaire de son discours et la construction d'un réseau utilitaire qui furent des facteurs de l'internationalisation de ses activités et de la modélisation de son action religieuse sur la scène locale et internationale. L'utilisation des technologies modernes a accéléré la mondialisation d'un modèle original de la da'wa. La trajectoire individualiste et transnationale de Ahmed Deedat montre bien que la mondialisation, loin de s'apparenter à un processus d'uniformisation culturelle et d'homogénéisation du monde, procède par réinvention de la différence et du local (Clifford, 1988 : 15).

Samadia SADOUNI

Wits Institute for Social and Economic Research (WISER), Johannesburg samsad48@hotmail.com 
Allievi Stefano, 2003, "Islam in the public space: social networks, media and neocommunities » in Allievi Stefano, Nielsen Jorgen S., Muslim Networks and Transnational Communities in and across Europe, Leiden, Brill, pp. 1-27.

Amiraux Valérie, 2001, Acteurs de l'islam entre Allemagne et Turquie : parcours militants et expériences religieuses, Paris, L'Harmattan.

Appadurai Arjun, 2001, Après le colonialisme. Les conséquences culturelles de la globalisation, Paris, Payot.

AsAD Talal, 1993, Genealogies of Religion: Discipline and Reasons of Power in Christianity and Islam, Baltimore, Johns Hopkins University Press.

Bennani-Chraï̈i Mounia, Fillieule Olivier (dir.), 2003, Résistances et protestations dans les sociétés musulmanes, Paris, Presses de Sciences Po.

Boltanski Luc et Chiapello Eve, 1999, Le nouvel esprit du capitalisme, Paris, Gallimard.

Castells Manuel, 1998, La société en réseaux. L'ère de l'information, Paris, Fayard.

CHAzel François, 1993, "La place du politique dans les mobilisations contestataires ", dans François Chazel, (dir.), Action collective et mouvements sociaux, Paris, Presses universitaires de France.

Clémentin-ojha Catherine et Gaborieau Marc, 1994, "La montée du prosélytisme dans le sous-continent indien ", Archives de sciences sociales des religions, 87, juilletseptembre 1994, pp. 13-33.

Clifford James, 1988, The Predicament of Culture. Twentieth Century Ethnography, Literature and Art, Cambridge (Mass.), Harvard University Press.

Colonomos Ariel, 1995, "Sociologie et science politique : les réseaux, théories et objets d'études ", Revue française de science politique, 45-1, février 1995, pp. 165-178.

Colonomos Ariel, 2000, Églises en réseaux. Trajectoires politiques entre Europe et Amérique, Paris, Presses de Sciences Po.

DeEDat Ahmed, Is the Bible God's World?, Durban, s.d.

DegenNe Alain, Forsé Michel, 1994, Les réseaux sociaux: une analyse structurale en sociologie, Paris, Armand Colin.

Esack Farid, 1997, Qur'an, Liberation and Pluralism. An Islamic Perspective of Interreligious Solidarity against Oppression, Oxford, Oneworld.

FourChard Laurent, MARY André et OtAYEK René, 2005, Entreprises religieuses et réseaux transnationaux en Afrique de l'Ouest, IFRA Ibadan, Karthala.

GuTWIRTH Jacques, 1998, L'église électronique : la saga des télévangélistes, Paris, Bayard.

HaEnni Patrick et Holtrop Tjitske, 2002, "Mondaines spiritualités... 'Amr Khâlid, "Shaykh" branché de la jeunesse dorée du Caire ", Politique africaine, 87, octobre 2002, pp. 45-68.

Hoeber Rudolph Susanne et Piscatori James (eds.), 1997, Transnational Religion and Fading States, Boulder (Co), Westview Press.

Ion Jacques (dir.), 2001, L'engagement au pluriel, Saint-Étienne, Publications de l'Université de Saint-Étienne.

Jamal Riaz Cassim, 1991, The Role and Contribution of the Islamic Propagation Centre International in the Field of Da'wah, Durban, Universite de Durban-Westville, non publié.

KASTORYANO Riva, 2000, "Immigrations, communautés transnationales et citoyenneté ", Revue internationale des sciences sociales, 165, septembre 2000. 
-, 1997, La France, l'Allemagne et leurs immigrés. Négocier l'identité, Paris, Armand Colin.

LubBe Gerrie J.A., 1987, "Muslims and Christians in South Africa ", Islamochristiana, 13, pp. 113-129.

MaMdani Mahmood, 2004, Citoyen et sujet: l'Afrique contemporaine et l'héritage du colonialisme tardif, Paris, Karthala.

Powell Avril A., 1993, Muslim and Missionaries in Pre-Munity India, Londres, Curzon Press.

RoberTSOn Roland, 1993, Globalization : Social Theory and Global Culture, Londres, Sage.

Roy Olivier, 2005, La laïcité face à l'islam, Paris, Stock.

SADOUNI Samadia, 1999, "Le minoritaire sud-africain Ahmed Deedat, une figure originale de la da'wa ", Islam et sociétés au sud du Sahara, 12, pp. 149-170.

-, 2000, "Les territoires d'un prédicateur musulman sud-africain ", dans FAure Véronique (dir.), Les dynamiques religieuses en Afrique Australe, Paris, Karthala, pp.161-173.

-, 1997, Islam " minoritaire ", diaspora et leadership en Afrique du Sud, Aix-en-Provence, Institut d'Études Politiques, non publié.

-, 2004, Minorités religieuses, intégrations, transnationalités : les «Indiens » musulmans de Durban, Afrique du Sud (1860-1994), Bordeaux, Université Montesquieu Bordeaux IV.

SHeIKH Naveed S., 2003, The new Politics of Islam: Pan-Islamic Policy in a World of States, Londres; New York, Routledge Curzon.

TAYob Abdulkader, 1995, Islamic Resurgence in South Africa. The Muslim Youth Movement, Le Cap, University of Cape Town Press.

Walls Andrew F., 1999, "Africa as the theatre of Christian engagement with Islam in the nineteenth century ", Journal of Religion in Africa, XXIX-2, pp. 155-174.

Westerlund David, 2003, "Ahmed Deedat's Theology of Religion : Apologetics through Polemics ", Journal of Religion in Africa, 33-3, pp. 263-278. 


\section{Résumé}

L'étude de la trajectoire transnationale du prédicateur-controversiste sud-africain d'origine indienne, Abmed Deedat, participe à l'analyse de l'individualisation et de la globalisation du mouvement de réislamisation depuis les années 1950. Le style original de son modèle de prédication islamique repose essentiellement sur la polémique islamo-chrétienne. Elle est présentée comme un nouveau mode de défense de l'islam face aux missionnaires chrétiens. Dans le contexte particulier de l'apartheid et de la "montée du prosélytisme" en Afrique, son discours traduit une affirmation identitaire qui vise la valorisation de l'islam face à un Occident perçu comme dominant. L'intégration dans des réseaux islamiques transnationaux de solidarité donne une dimension internationale au prédicateur sud-africain qui n'est pas sans évoquer les techniques de mobilisation des télévangélistes américains.

Mots-clés : prédicateur, polémique religieuse, communauté, transnational, réseau.

\section{Abstract}

The study of the transnational trajectory of the South African preacher of Indian origin, Abmed Deedat, takes part in the analysis of the individualisation in course in the movement of reislamisation since the 1950's and its relationships to the globalization. The original style of his model of Islamic preaching rests primarily on religious polemics which is presented as a new mode of religious action in the defence of Islam against the Christian missionaries. In the specific context of the apartheid and "rise of proselytism" in Africa, his discourse reveals an identity assertion which aims at the apologetics of Islam faced with the Occident perceived as hegemonic. His integration within transnational islamic networks of solidarity enables the South African preacher to give an international dimension to his career which singularly evoke the modes of mobilization of the American TV evangelists.

Key words: Preacher, religious controversy, community, transnational, network.

\section{Resumen}

El estudio de la trayectoria transnacional del predicador-polemista sudafricano de origen indio, Abmed Deedat, participa del análisis de la individuación en curso en el movimiento de reislamización desde los años 1950 y de sus relaciones con la globalización. El estilo original de su modelo de prédica islámica reposa esencialmente en la polémica islámico-cristiana, que es presentada como un nuevo modo de acción religiosa en la defensa del Islam frente a los misioneros cristianos. En el contexto particular del apartheid y del "crecimiento del proselitismo" en África, su discurso traduce una afirmación identitaria que apunta a la valorización del Islam frente a un Occidente percibido como dominante. La integración a redes islámicas de solidaridad en el espacio transnacional dará una dimensión internacional al predicador sudafricano cuyas estrategias internacionalistas evocan singularmente a los modos de movilización de los televangelistas americanos. (Trad. de Véronica Béliveau-Giménez)

Palabras clave : predicador, polémica religiosa, comunidad, transnacional, red. 